\title{
Food Cues Do Not Modulate the Neuroendocrine Response to a Prolonged Fast in Healthy Men
}

\author{
Marieke Snel $^{a} \quad$ Marjolein A. Wijngaarden $^{b}$ Maurice B. Bizino ${ }^{a}$ \\ Jeroen van der Grond ${ }^{c}$ Wouter M. Teeuwisse ${ }^{c}$ Mark A. van Buchem ${ }^{c}$ \\ Ingrid M. Jazet ${ }^{\mathrm{a}}$ Hanno Pijl ${ }^{\mathrm{b}}$
}

Departments of a General Internal Medicine, ${ }^{\mathrm{b}}$ Endocrinology and Metabolism, and ${ }^{\mathrm{c}}$ Radiology, Leiden University

Medical Center, Leiden, The Netherlands

\section{Key Words}

Starvation · Glucose tolerance $\cdot$ Functional magnetic

resonance imaging $\cdot$ Hypothalamus

\begin{abstract}
Introduction: Dietary restriction benefits health and increases lifespan in several species. Food odorants restrain the beneficial effects of dietary restriction in Drosophila melanogaster. We hypothesized that the presence of visual and odorous food stimuli during a prolonged fast modifies the neuroendocrine and metabolic response to fasting in humans. Subjects and Methods: In this randomized, crossover intervention study, healthy young men $(n=12)$ fasted twice for $60 \mathrm{~h}$; once in the presence and once in the absence of food-related visual and odorous stimuli. At baseline and on the last morning of each intervention, an oral glucose tolerance test (OGTT) was performed. During the OGTT, blood was sampled and a functional MRI scan was made. Results: The main effects of prolonged fasting were: (1) decreased plasma thyroid stimulating hormone and triiodothyronine levels; (2) downregulation of the pituitary-gonadal axis; (3) reduced plasma glucose and insulin concentrations, but increased glucose and insulin responses to glucose ingestion;
\end{abstract}

(4) altered hypothalamic blood oxygenation level-dependent (BOLD) signal in response to the glucose load (particularly during the first 20 min after ingestion); (5) increased resting energy expenditure. Exposure to food cues did not affect these parameters. Conclusion: This study shows that $60 \mathrm{~h}$ of fasting in young men (1) decreases the hypothalamic BOLD signal in response to glucose ingestion; (2) induces glucose intolerance; (3) increases resting energy expenditure, and (4) downregulates the pituitary-thyroid and pituitary-gonadal axes. Exposure to visual and odorous food cues did not alter these metabolic and neuroendocrine adaptations to nutrient deprivation.

Copyright $\odot 2012$ S. Karger AG, Basel

\section{Introduction}

Dietary restriction extends life span and prevents prevalent age-related diseases like cancer and diabetes in a variety of species, including nonhuman primates [1]. The benefits appear to come about at least in part through

M.S. and M.A.W. contributed equally to this work.

\section{KARGER}

Fax +4161306 1234

E-Mail karger@karger.ch

www.karger.com
(C) 2012 S. Karger AG, Basel

0028-3835/12/0964-0285\$38.00/0

Accessible online at:

www.karger.com/nen
Department of Endocrinology and Metabolism (C4-82)

Leiden University Medical Center, PO Box 9600

NL-2300 RC Leiden (The Netherlands)

Tel. +31 715263 793, E-Mail h.pijl@lumc.nl 
metabolic and neuroendocrine adaptations to nutrient deprivation [2]. Circumstantial evidence suggests that dietary restriction may have similar effects on health and longevity in humans, probably at least in part via established endocrine and metabolic adaptations to nutrient deprivation [1].

It has recently been shown that food odorants (from live yeast) restrain the beneficial effects of dietary restriction on longevity in Drosophila melanogaster [3]. Moreover, mutation of a gene leading to severe olfactory defects (Orb83b) alters metabolism, enhances stress resistance and extends life span in fully fed Drosophila [3], corroborating other evidence that aging and longevity are regulated by olfactory neurons in Caenorhabditis elegans [4].

The vomeronasal organ (VNO) is an evolutionary conserved part of the (mammalian) olfactory system, which primarily responds to nonvolatile cues to relay environmental information to the hypothalamus (in mammals), allowing subsequent adaptation of reproductive and ingestive behavior and neuroendocrine secretion [5]. The VNO primarily transmits pheromonal signals, mediating social and sexual behaviors and neuroendocrine changes pertaining to reproduction. Limited evidence indicates that (non-pheromonal) odorous stimuli perceived by the main olfactory epithelium (MOE) can also engage hypothalamic neurons in humans [6]. The hypothalamus controls systemic glucose and lipid flux in response to circulating metabolic and hormonal cues reflecting bodily energy reserves [7]. Visual food cues also impact on hypothalamic neuronal activity in healthy humans [8], suggesting that this type of stimulus may add to the putative effects of odors on neuroendocrine and metabolic features in the current experimental context.

Here we hypothesized that visual and odorous food stimuli impact on neuroendocrine and metabolic responses to a prolonged fast in humans. Specifically, we predict that exposure to attractive visual and odorous food cues during fasting blunts the hypothalamic and metabolic adaptations that normally occur in response to nutrient deprivation. Although we realize that fasting is not an ideal model of chronic (mild) restriction of calories, we use (prolonged) fasting as a proxy, since randomized offering of food cues to people who restrict their calories for a long time is virtually impossible. Moreover, we reasoned that if odorous cues counteract the benefits of mild restriction, they may impact the corollaries of more severe restriction to an even greater extent. We quantify neuronal activity in the hypothalamus by functional magnetic resonance imaging (fMRI); this technique has previously been used to determine hypothalamic activity in response to glucose ingestion in humans [9-11]. We chose to use total fasting instead of (prolonged) calorie restriction as a model of nutrient deprivation to maximize the endocrine effects of short term deprivation, since longer-term experiments of this kind are not feasible in humans, particularly with respect to modulation of exposure to food cues. Visual food stimuli were offered in addition to odorous cues to maximize the potential impact of exposure.

\section{Subjects and Methods}

\section{Subjects}

This study was executed in accordance with the principles of the revised Declaration of Helsinki and commenced after approval of the Medical Ethics Committee of the Leiden University Medical Center. This trial was registered at Clinicaltrials.gov (NCT01243879). All volunteers gave written informed consent before participation. We studied 12 healthy Caucasian males who were recruited via local advertisements. All participants were between 18 and 30 years (mean 22 years), had a body mass index (BMI) ranging from 20-25 (mean 22.5) and had fasting serum glucose levels below $6.1 \mathrm{mmol} / \mathrm{l}$. Subjects who used medication, smoked, suffered from anosmia, had MRI contraindications or had recently donated blood were excluded from participation.

\section{Study Design}

In this randomized, controlled, crossover intervention study, participants were exposed to two sequential interventions that consisted of $60 \mathrm{~h}$ of fasting in the presence or absence of foodrelated stimuli. Interventions occurred in random order with a washout period of at least 2 weeks. In the week before the first intervention, after an overnight fast, the baseline fMRI scan and oral glucose tolerance test (OGTT) were performed.

On a separate day the next week, subjects were admitted to our clinical research center at 08:00 a.m. after an overnight fast (12 h) for a subsequent period of $48 \mathrm{~h}$ of fasting. After arrival, anthropometric measures were taken and substrate oxidation was measured by indirect calorimetry. During both interventions, volunteers were allowed to drink water ad libitum, but were not permitted to leave the research center. During the intervention without food-related cues, participants were not allowed to talk about food or to watch food-related matters on television.

During the other intervention, subjects were exposed to visual and odorous food stimuli food from 10:00 a.m. to 12:30 p.m. (eggs with bacon, grilled sandwich and coffee), from 1:00 to 3:30 p.m. (apple pie and banana cake), from 5:00 to 8:30 p.m. (French fries with meat croquettes, pizza salami and garlic bread) and from 9:00 to 10:00 p.m. (coffee). All food items were freshly prepared and presented to the participants. On the last morning, food stimuli were given from 6:00 to 7:00 a.m. (bacon, grilled sandwich and coffee).

After each 60-hour fast (with or without exposure to visual and odorous food stimuli), an OGTT and fMRI scanning were performed simultaneously. At 7:00 a.m. an intravenous catheter was 
inserted. Subjects drank $75 \mathrm{~g}$ of glucose dissolved in $300 \mathrm{ml}$ of water at $\mathrm{t}=0$. Blood samples were drawn at $\mathrm{t}=-15,0,15,30,45,60$, $75,90,105$ and $120 \mathrm{~min}$. During the OGTT, hypothalamic neuronal activity was measured by fMRI from time -8 to $+28 \mathrm{~min}$.

Anthropomorphic Measurements, Blood Pressure, Heart Rate

Height, weight, BMI, hip and waist circumference were measured according to WHO recommendations. Blood pressure was measured automatically (Omron 705IT, Kyoto, Japan) at the left arm with the subject in supine position after 5 min of rest. Heart rate was measured by palpation of a radial artery during $1 \mathrm{~min}$ (in rest).

\section{Indirect Calorimetry}

Subjects were placed under the ventilated hood after 12 and 60 h of fasting (Oxycon Beta, Mijnhardt Jaegher, Breda, The Netherlands). After voiding, the patient had to lie still with eyes closed, while staying awake, for $30 \mathrm{~min}$. Substrate oxidation was calculated from $\mathrm{CO}_{2}$ and $\mathrm{O}_{2}$ concentrations in the exhaled air as previously described [12]. If a calculated substrate oxidation value was below 0 , we used 0 instead for our statistical analysis.

\section{$M R I$}

fMRI scans were made using a 3.0-Tesla scanner (Philips Achieva; Philips Healthcare, Best, The Netherlands). A $14 \mathrm{~mm}$ thick midsagittal brain slice was imaged for a total time of 38.2 min by $\mathrm{T}_{2}{ }^{*}$-weighted echo-planar imaging (repetition time 120 $\mathrm{ms}$, echo time $30 \mathrm{~ms}$, flip angle $30^{\circ}$, scan matrix $256 \times 231$, FOV $208 \times 208 \times 14 \mathrm{~mm}, 900$ dynamics). We used a multishot EPI sequence with an EPI factor of 33, with 3 signal averages (nsa). No parallel imaging was used. To diminish rotations during the scanning procedure, each participant's head was fixated with cushions inside the coil.

\section{MRI Analysis}

fMRI data were analyzed using FSL software (FMRIB's Software Library, www.fmrib.ox.ac.uk/fsl) $[13,14]$. First, imaginary and real images were calculated from the 900 magnitude and corresponding phase images that were acquired. Next, for each participant separately all magnitude images were registered (aligned) to a dynamic image that was acquired shortly after drinking had finished and that showed no motion artifacts (MCFLIRT) [15]. The resulting transformation matrix was then used to register the real and imaginary images (FLIRT). After complex averaging of every 4 images, the real and imaginary data were converted back to magnitude and phase images, resulting in 225 magnitude images to be analyzed. A region of interest was manually drawn in the hypothalamic area to calculate (with the 'fslmeants' command) the average hypothalamic signal for all 225 time points. We used the anterior commissure, the mammillary bodies and the optic chiasm as border 'points' of the hypothalamic region of interest. The fourth border point was estimated on the virtual square resulting from the three defined points. After drawing the regions of interest, an average was created for each minute that scanning was performed. The signal of each time point was normalized to the averaged pre-drink signal, rendering the relative signal change. The investigator was not blind to the occasion when analyzing the fMRI data, but the only subjective (i.e. not automated) analytical procedure was the definition of the hypothalamic region. Moreover, all analyses were performed twice to minimize observer bias.

\section{Blood Chemistry}

Serum glucose was measured using a Modular P800 chemistry analyzer by Roche Diagnostics (Mannheim, Germany) with a total coefficient of variation $(\mathrm{CV})$ of $1.7 \%$. Insulin was measured with an immunometric assay on an automated Immulite 2500 (Siemens, Breda, The Netherlands) with an intra-assay CV of 6-7.5\%. Serum cholesterol, high-density lipoprotein (HDL) and triglycerides (TG) were measured with a fully automated P-800 module (Roche, Almere, The Netherlands). For both TG and total cholesterol (TC) the CV was less than $2 \%$. For HDL the CV was less than 3\%. Low-density lipoprotein (LDL) cholesterol was calculated according to the Friedewald equation. Prolactin, luteinizing hormone ( $\mathrm{LH}$ ) and follicle-stimulating hormone (FSH) were measured using an electrochemoluminescence immunoassay (ECLIA) on a Modular E170 analyzer by Roche Diagnostics. Total CVs were less than 3.5\%. Serum growth hormone (GH) was measured with a sensitive immunofluorometric assay (Wallac, Turku, Finland), specific for the $22-\mathrm{kDa}$ GH protein, calibrated against World Health Organization International Reference Preparation (WHO IRP) 80/505. The detection limit is $0.03 \mathrm{mU} / \mathrm{l}$, the interassay CV between 2 and $9 \%$ for concentrations from $0.25-40 \mathrm{mU} / \mathrm{l}$. Serum insulin-like growth factor 1 (IGF-1) was measured using an immunometric technique on an Immulite 2500 system (Diagnostic Products Corporation, Los Angeles, Calif., USA). The intra-assay CVs were 5.0 and $7.5 \%$ at mean plasma levels of 8 and 75 $\mathrm{nmol} / \mathrm{l}$, respectively.

Insulin-like growth factor binding protein 3 (IGFBP-3) was measured using an immunometric technique on an Immulite 2500 system (Diagnostic Products Corporation, Los Angeles, Calif., USA). The lower limit of detection was $0.02 \mathrm{mg} / \mathrm{l}$ and interassay variation was 4.4 and $4.8 \%$ at 0.91 and $8.83 \mathrm{mg} / \mathrm{l}$, respectively.

Serum free T4 (FT4) and thyroid stimulating hormone (TSH) were measured using a chemoluminescence immunoassay with a Modular Analytics E-170 system (Roche, Almere, The Netherlands). The intra-assay CVs were $1.6-2.2 \%$ and $1.3-5.0 \%$, respectively. Serum triiodothyronine (T3) was measured with a fluorescence polarization immunoassay on an AxSym system (Abbott, Abbott Park, Ill., USA). The CV was 2.5-9.0\%.

Dehydroepiandrosterone (DHEA) was measured by radioimmunoassay (RIA) (DSL, a Beckman Coulter Company). The detection limit was $0.012 \mu \mathrm{g} / \mathrm{l}(0.04 \mathrm{nmol} / \mathrm{l})$; the intra-assay CV was between 5.2 and $10.8 \%$, the interassay CV between 5.9 and $11.7 \%$.

Testosterone was determined by a direct RIA by Siemens Healthcare Diagnostics; total CV was approximately $15 \%$.

Sex hormone-binding globulin (SHBG) was measured on an automated Immulite 2500 (Siemens, Breda, The Netherlands) with a total CV of $8 \%$.

Cortisol was measured using a chemoluminescence immunoassay on a Modular Analytics E-170 system (Roche, Almere, The Netherlands). Total CV was less than $3.5 \%$ for levels between 0.19 and $1.08 \mu \mathrm{mol} / \mathrm{l}$.

\section{Statistics}

The differences between values before and after interventions were statistically evaluated by two-sided paired Student's t tests. The impact of food cues on the response to nutrient deprivation was evaluated by subtracting values obtained after intervention from those obtained before, yielding delta values. The difference between deltas observed during fasting with versus without stimuli was subsequently evaluated by two-sided paired Student's t 
Table 1. Anthropomorphic and cardiovascular measurements

\begin{tabular}{lcccc}
\hline & $\begin{array}{l}\text { Before starvation } \\
\text { without stimuli }\end{array}$ & $\begin{array}{l}\text { After starvation } \\
\text { without stimuli }\end{array}$ & $\begin{array}{l}\text { Before starvation } \\
\text { with stimuli }\end{array}$ & $\begin{array}{l}\text { After starvation } \\
\text { with stimuli }\end{array}$ \\
\hline Age, years & $22.3 \pm 0.8$ & & & \\
Length, cm & $183 \pm 2$ & & & $72.8 \pm 2.1^{*}$ \\
Body weight, kg & $75.8 \pm 2.3$ & $73.0 \pm 2.2^{*}$ & $75.8 \pm 2.1$ & $21.6 \pm 0.5^{*}$ \\
BMI & $22.5 \pm 0.5$ & $21.7 \pm 0.5^{*}$ & $22.5 \pm 0.5$ & $67 \pm 4$ \\
Heart rate, bpm & $76 \pm 4$ & $69 \pm 3$ & $66 \pm 3$ & $1.4 \pm 3.8^{* *}$ \\
Delta heart rate, bpm & $139 \pm 4$ & $-7.3 \pm 3.9$ & $143 \pm 6$ \\
Systolic blood pressure, $\mathrm{mm} \mathrm{Hg}$ & $78 \pm 2$ & $76 \pm 2$ & $77 \pm 2$ & $73 \pm 2$ \\
Diastolic blood pressure, $\mathrm{mm} \mathrm{Hg}$ & & & & $138 \pm 4$ \\
\hline
\end{tabular}

Data are depicted as mean \pm SEM (standard error of the mean).

* Significantly $(\mathrm{p}<0.05)$ different before and after the starvation intervention. ${ }^{* *}$ Significantly $(\mathrm{p}<0.05)$ different between the two starvation paradigms.

There are no significant differences between the different (with and without stimuli) starvation paradigms.

test. Blood oxygenation level-dependent (BOLD) signals were averaged per minute and subsequently evaluated statistically by repeated measures ANOVA and post hoc tested with pairwise comparisons. All statistics were performed with SPSS for Windows version 16.0 (SPSS Inc., Chicago, Ill. USA).

\section{Results}

\section{Anthropometric Measurements, Heart Rate and Blood} Pressure

Body weight, BMI, hip circumference and lean body mass were diminished to a similar extent by the prolonged fast with and without exposure to food-related stimuli (table 1). Heart rate diminished significantly during fasting without stimuli, whereas heart rate increased when food-related stimuli were given during the fast (delta heart rate $-7.3 \pm 3.9$ after fasting without stimuli vs. $1.4 \pm 3.8$ after fasting with stimuli, $\mathrm{p}=0.041$ ). The prolonged fast did not significantly alter blood pressure (table 1).

\section{Lipids and Hormones in Plasma}

Plasma levels of cholesterol, TG and the cholesterol/ HDL ratio significantly increased, whereas HDL-cholesterol decreased significantly and equally after $60 \mathrm{~h}$ of food abstinence with or without stimuli. LDL-cholesterol only increased significantly after fasting without stimuli. Plasma TSH and T3 levels decreased, whereas T4 levels remained unchanged in response to the prolonged fast (table 2). Plasma levels of LH, FSH and testosterone de- creased significantly, whereas SHBG levels increased (table 2).

Prolactin, cortisol and IGFBP-3 levels remained unaltered. The concentrations of 2 hormones were altered significantly only in response to fasting without stimuli: DHEA levels increased, whereas IGF-1 levels decreased in this experimental context. However, the difference in response between fasting with and without food cues did not reach statistical significance for either hormone.

\section{OGTT}

Plasma glucose and insulin concentrations reduced considerably upon prolonged fasting (table 3). In contrast, the fast significantly increased the areas under the curves of plasma glucose and insulin concentrations in response to glucose ingestion (fig. 1). The times to peak of plasma glucose and insulin concentrations were delayed by nutrient deprivation. Exposure to odorous food cues during the prolonged fast did not affect any of these metabolic adaptations.

\section{$f M R I$}

The hypothalamic BOLD signal, expressed as percentage of the averaged pre-drink values, was reduced in response to glucose ingestion compared to baseline (at several time points to a significant extent, particularly during the first $20 \mathrm{~min}$ after ingestion), irrespective of exposure to food cues during the prolonged fast (fig. 2). The prolonged fast with stimuli induced significant differences compared to baseline at $\mathrm{t}=4(\mathrm{p}<$ $0.001), 9(\mathrm{p}=0.033), 16(\mathrm{p}=0.025), 20(\mathrm{p}=0.025)$ and 27 
Table 2. Plasma metabolites and hormones at baseline and after the interventions

\begin{tabular}{lcccc}
\hline & $\begin{array}{c}\text { Before starvation } \\
\text { without stimuli }\end{array}$ & $\begin{array}{c}\text { After starvation } \\
\text { without stimuli }\end{array}$ & $\begin{array}{c}\text { Before starvation } \\
\text { with stimuli }\end{array}$ & $\begin{array}{l}\text { After starvation } \\
\text { with stimuli }\end{array}$ \\
\hline Total cholesterol, mmol/l & $4.22 \pm 0.18$ & $4.42 \pm 0.20^{*}$ & $4.25 \pm 0.18$ & $4.39 \pm 0.19$ \\
Triglycerides, mmol/l & $0.94 \pm 0.08$ & $1.33 \pm 0.11^{*}$ & $0.98 \pm 0.13$ & $1.36 \pm 0.11^{*}$ \\
HDL, mmol/l & $1.42 \pm 0.08$ & $1.25 \pm 0.07^{*}$ & $1.43 \pm 0.06$ & $1.25 \pm 0.06^{*}$ \\
Cholesterol/HDL & $3.06 \pm 0.19$ & $3.63 \pm 0.19^{*}$ & $3.05 \pm 0.22$ & $3.58 \pm 0.23^{*}$ \\
LDL, mmol/l & $2.38 \pm 0.15$ & $2.58 \pm 0.19^{*}$ & $2.38 \pm 0.21$ & $2.54 \pm 0.21$ \\
FT4, nmol/l & $16.53 \pm 0.60$ & $16.60 \pm 0.70$ & $16.67 \pm 0.37$ & $17.18 \pm 0.60$ \\
T3, nmol/l & $1.82 \pm 0.08$ & $1.14 \pm 0.08^{*}$ & $1.91 \pm 0.06$ & $1.28 \pm 0.04^{*}$ \\
TSH, mU/l & $2.92 \pm 0.54$ & $1.26 \pm 0.19^{*}$ & $2.47 \pm 0.37$ & $1.25 \pm 0.18^{*}$ \\
Prolactin, $\mu \mathrm{g} / \mathrm{l}$ & $13.4 \pm 1.25$ & $14.18 \pm 2.79$ & $10.93 \pm 1.66$ & $11.23 \pm 1.59$ \\
DHEA, nmol/l & $10.20 \pm 0.78$ & $12.30 \pm 1.26^{*}$ & $11.99 \pm 1.56$ & $12.88 \pm 1.31$ \\
Human GH, mU/l & $4.63 \pm 3.25$ & $11.74 \pm 5.19$ & $2.11 \pm 1.23$ & $7.97 \pm 2.38^{* *}$ \\
IGF-1, nmol/l & $27.30 \pm 1.46$ & $23.01 \pm 1.40^{*}$ & $25.05 \pm 2.99$ & $23.79 \pm 1.59$ \\
IGFBP-3, mg/l & $4.32 \pm 0.18$ & $4.33 \pm 0.31$ & $6.72 \pm 2.74$ & $4.18 \pm 0.28$ \\
Cortisol, $\mu \mathrm{mol} / \mathrm{l}$ & $0.51 \pm 0.03$ & $0.55 \pm 0.04$ & $0.53 \pm 0.04$ & $0.49 \pm 0.05$ \\
FSH, U/l & $4.0 \pm 0.6$ & $3.2 \pm 0.5^{*}$ & $4.1 \pm 0.6$ & $3.3 \pm 0.5^{*}$ \\
LH, U/l & $5.0 \pm 0.3$ & $3.3 \pm 0.4^{*}$ & $5.1 \pm 0.2$ & $3.2 \pm 0.4^{*}$ \\
SHBG, nmol/l & $25.3 \pm 1.9$ & $28.3 \pm 2.1^{*}$ & $24.7 \pm 1.8$ & $28.0 \pm 1.7^{*}$ \\
Testosterone, nmol/l & $28 \pm 2.5$ & $14.4 \pm 1.8^{*}$ & $27 \pm 1.6$ & $14.7 \pm 1.3^{*}$ \\
\hline
\end{tabular}

Data are depicted as mean \pm SEM (standard error of the mean).

* Significantly $(\mathrm{p}<0.05)$ different before and after the starvation intervention. ${ }^{* *} \mathrm{p}=0.06$.

There are no significant differences between the different (with and without stimuli) starvation paradigms.

Table 3. Results of OGTT: measurements of glucose, insulin and C-peptide at baseline and after the interventions

\begin{tabular}{lccc}
\hline & Baseline $^{\mathrm{a}}$ & $\begin{array}{l}\text { Starvation without stimuli } \\
\text { after intervention }\end{array}$ & $\begin{array}{c}\text { Starvation with stimuli } \\
\text { after intervention }\end{array}$ \\
\hline Glucose, mmol/l & $5.20 \pm 0.09$ & $3.69 \pm 0.13^{*}$ & $3.67 \pm 0.10^{*}$ \\
Insulin, mU/l & $4.33 \pm 0.56$ & $2.33 \pm 0.19^{*}$ & $2.08 \pm 0.08^{*}$ \\
AUC glucose & $1,068 \pm 44$ & $1,481 \pm 51^{* *}$ & $1,455 \pm 41^{* *}$ \\
AUC insulin & $3,959 \pm 312$ & $8,472 \pm 1,376^{* *}$ & $8,042 \pm 1,202^{* *}$ \\
Time to peak glucose & $51 \pm 8$ & $101 \pm 8^{*}$ & $104 \pm 7^{*}$ \\
Time to peak insulin & $75 \pm 9$ & $113 \pm 6^{*}$ & $120 \pm 6^{*}$ \\
\hline
\end{tabular}

Data are depicted as mean \pm SEM (standard error of the mean).

${ }^{a}$ As measured at screening after an overnight fast. ${ }^{*}$ Significantly $(\mathrm{p}<0.025)$ different from baseline. ${ }^{*}$ Significantly $(\mathrm{p}<0.01)$ different from baseline.

There are no significant differences between the different (with and without stimuli) starvation paradigms.

$\min (\mathrm{p}=0.031)$. The prolonged fast without stimuli induced significant differences when compared to baseline at $\mathrm{t}=4 \min (\mathrm{p}=0.012)$, with trends at $\mathrm{t}=7(\mathrm{p}=$ $0.053), 20$ ( $\mathrm{p}=0.060), 25$ ( $\mathrm{p}=0.056), 26 \mathrm{~min}(\mathrm{p}=0.055)$. Repeated analysis confirmed these results (data not shown).

Food Cues and Response to Fasting in Humans

\section{Indirect Calorimetry}

Prolonged fasting significantly decreased glucose oxidation and increased lipid oxidation. There were no significant differences between the fasting paradigms (table 4). Prolonged fasting increased resting energy expenditure significantly and consistently, irrespective of the exposure to food cues. 


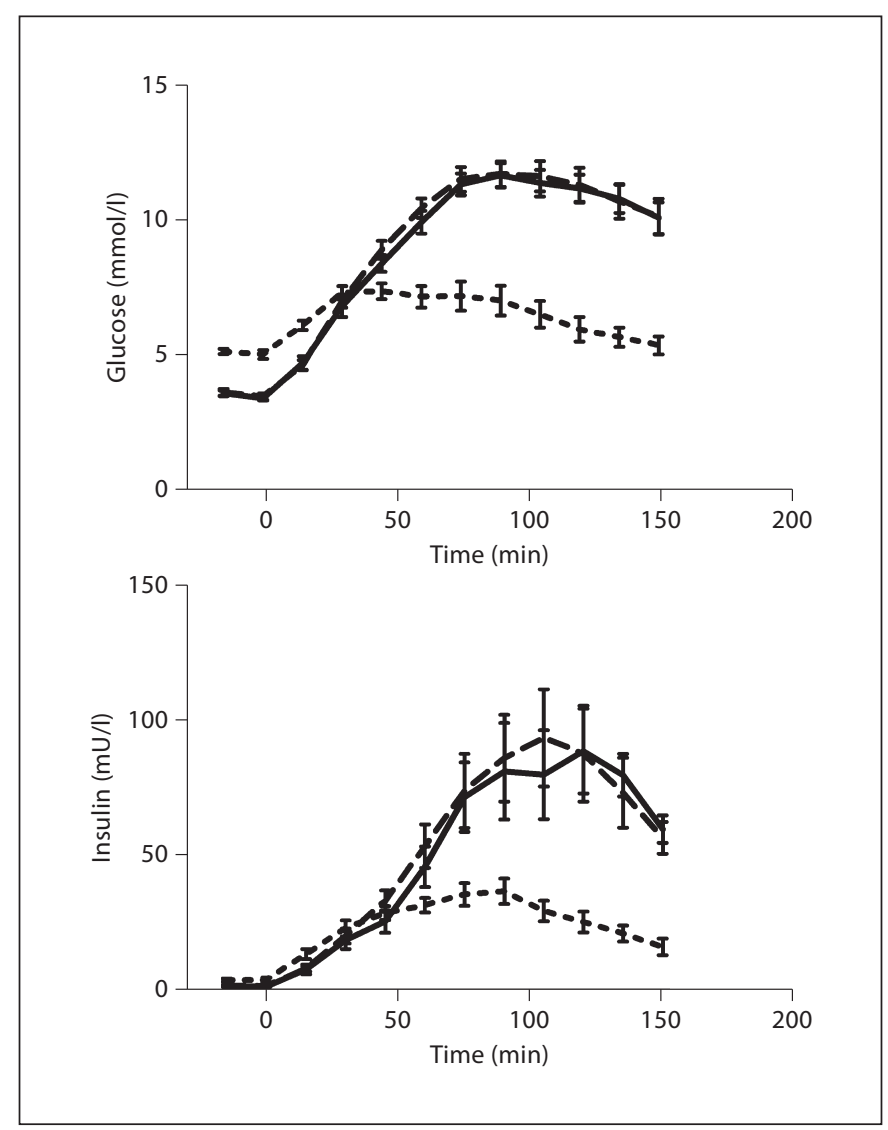

Fig. 1. Plasma glucose and insulin concentrations in response to an oral glucose load at baseline and after the interventions. The dotted line represents baseline levels, the dashed line represents levels after the prolonged fast without stimuli and the continuous line represents levels after the prolonged fast with stimuli.

\section{Discussion}

This study shows that 60 hours of food deprivation in healthy young men (1) alters the hypothalamic BOLD signal in response to glucose ingestion; (2) induces profound glucose intolerance; (3) downregulates the pituitary-thyroid and pituitary-gonadal axes, and (4) increases resting metabolic rate. Exposure to (attractive) visual and odorous food cues does not alter any of these metabolic and neuroendocrine adaptations to nutrient deprivation.

This is the first study to show that prolonged fasting alters the BOLD signal produced by the hypothalamus in response to an oral glucose load in healthy humans. Since BOLD signals reflect neuronal activity [16], the data suggest that a prolonged fast renders the hypothalamic neurons relatively insensitive to glucose ingestion. It is con-

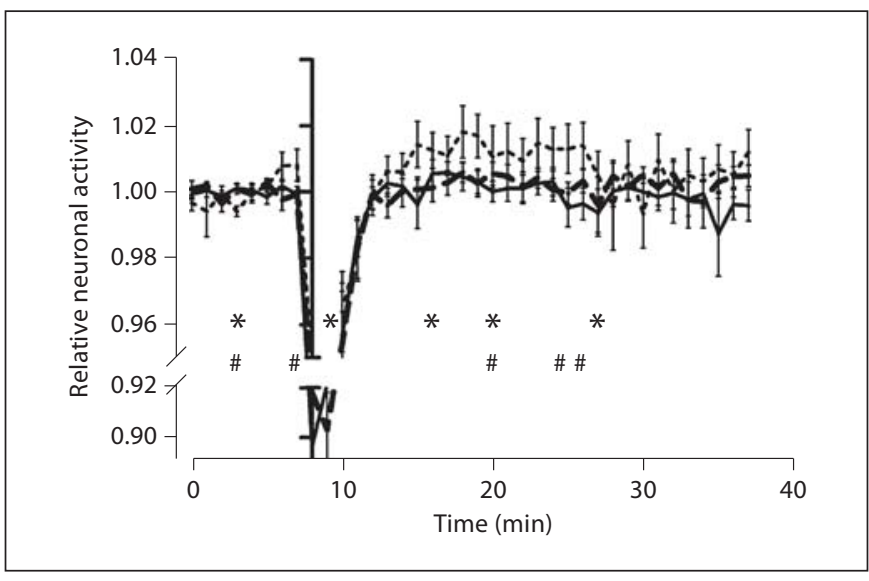

Fig. 2. Hypothalamic neuronal activity in response to glucose intake at $\mathrm{t}=0 \mathrm{~min}$ as measured by fMRI. Relative neuronal hypothalamic activity measured before and after the OGTT (started at $\mathrm{t}=8 \mathrm{~min}$ ), percent change to pre-drink average is depicted. ${ }^{*}$ The prolonged fast with stimuli (continuous line) showed significant differences when compared to baseline (dotted line) at $\mathrm{t}=4(\mathrm{p}=$ $0.000), 9(\mathrm{p}=0.033), 16(\mathrm{p}=0.025), 20(\mathrm{p}=0.025)$ and $27 \mathrm{~min}$ $(\mathrm{p}=0.031)$. \# The prolonged fast without stimuli (dashed line) showed significant differences when compared to baseline (dotted line $)$ at $t=4 \min (p=0.012)$, with trends at $t=7(p=0.053)$, $20(\mathrm{p}=0.060), 25(\mathrm{p}=0.056)$ and $26 \mathrm{~min}(\mathrm{p}=0.055)$.

ceivable that alterations of hypothalamic neuronal activity are an integral part of the systemic adaptations required for survival during nutrient deprivation. The hypothalamus plays a critical role in the control of (postprandial) metabolism; it integrates metabolic and hormonal cues reflecting the bodily energy status to produce neuroendocrine output, adapting energy metabolism accordingly [17]. For example, neuropeptide Y (NPY, a wellknown orexigenic neuropeptide) gene expression is strongly upregulated in the arcuate nucleus and paraventricular nucleus of fasting rhesus macaque monkeys and food-restricted type I diabetic rats $[18,19]$. Hypothalamic NPY induces hepatic insulin resistance via activation of sympathetic neural efferents [20]. Other hypothalamic neuronal circuits are also sensitive to nutritional cues and contribute to proper (postprandial) metabolic control [21]. Therefore, the neuronal changes in response to nutrient deprivation we observed here may be involved in the physiology of the glucose intolerance of our fasting volunteers. Insulin resistance is an appropriate metabolic adaptation to nutrient deprivation, as it renders incidentally consumed glucose available for combustion by the brain (which is largely dependent on glucose as fuel). Since nutrients tend to modulate hypothalamic neural 
Table 4. Substrate oxidation rates at baseline and after 3 days of starvation with or without the presence of food-related stimuli

\begin{tabular}{|c|c|c|c|c|}
\hline Glucose oxidation, $\mu \mathrm{mol} / \mathrm{kg}_{\mathrm{FFM}} / \mathrm{min}$ & $12.3 \pm 1.5$ & $7.9 \pm 2.3^{* *}$ & $12.6 \pm 2.6$ & $4.0 \pm 1.0^{*}$ \\
\hline Resting energy expenditure, $\mathrm{kcal} / \mathrm{day}$ & $1,631 \pm 51$ & $1,829 \pm 71^{*}$ & $1,696 \pm 51$ & $1,895 \pm 103^{*}$ \\
\hline Resting energy expenditure, kcal/day/LBM & $25.6 \pm 0.7$ & $30.5 \pm 0.9^{*}$ & $27.2 \pm 0.7$ & $31.9 \pm 1.3^{*}$ \\
\hline
\end{tabular}

circuits so as to reinforce insulin action [22], resistance to the neuronal effects of nutrient ingestion may be advantageous in terms of brain energetics and survival during prolonged periods of food deprivation.

The greatest effect of the oral glucose tolerance test on plasma values of glucose and insulin are seen at 50 and $75 \mathrm{~min}$ after glucose ingestion, respectively, whereas fMRI signals respond within 10-20 min (fig. 1,2). These observations suggest that neither plasma glucose nor insulin is involved in the neuronal response to glucose ingestion. This inference is supported by previous work suggesting that neuronal signals emanating from the gut rather than plasma metabolite concentrations are critical for the hypothalamic response to nutrient ingestion [23].

Although we have used fMRI to quantify hypothalamic neuronal activity in similar experimental settings before [11], it seems important to note that the use of this imaging technique to measure neuronal activity in response to a single (non-iterative) stimulus is relatively uncommon and bears some difficulties. Perhaps the most important pitfall in our analysis is the rather subjective determination of the region of interest (designating the hypothalamus in the current study): small differences may significantly impact the average signal. To preclude observer bias as much as possible, the same author (M.A.W.) performed all analyses of BOLD signal changes twice, yielding similar results.

The fall of circulating T3 and TSH levels in the face of relatively stable free T4 concentrations are typical physiological adaptations of the pituitary-thyroid axis to shortterm fasting in humans $[24,25]$. These changes, which may serve to dampen basal energy expenditure, partly result from an adaptive mechanism driven by diminished activity of leptin-sensitive hypothalamic thyrotropin-releasing hormone neurons [25]. However, despite these apparent effects of nutrient deprivation on thyroid axis ac-

Food Cues and Response to Fasting in Humans tivity, $60 \mathrm{~h}$ of fasting paradoxically increased resting energy expenditure by $\sim 11 \%$ in our volunteers. We are not the first to report this counterintuitive observation: a few previous studies similarly showed that the resting metabolic rate (RMR) of healthy humans is increased by 6 and $4 \%$ after 36 or 48 h of food deprivation, respectively [24, 26]. The increase in RMR during short-term fasting might be due to the energy costs of a temporary increase in gluconeogenesis and ketogenesis [24]. Activation of the sympathetic nervous system may modify RMR during prolonged fasting [27]. In apparent contrast, RMR is clearly reduced after longer-term calorie restriction and weight loss in obese humans [28], but this is probably due to the significant loss of lean body mass in this context [29].

Plasma levels of FSH, LH and testosterone significantly decreased upon fasting, while SHBG concentrations increased, which confirms the findings of other studies [30]. Long-term dietary restriction over the course of years also downregulates the pituitary-gonadal axis in members of the Calorie Restriction Society [31]. These endocrine adaptations probably serve to postpone reproduction until food is available again. Reduction of plasma leptin levels may be mechanistically involved, since leptin administration during a prolonged fast prevents changes in testosterone levels (but not LH and SHBG) in healthy men [25].

We also show that fasting increases circulating DHEA levels (significantly in the group that was not exposed to food-related stimuli). DHEA and its sulfate ester DHEAS are markers of human aging, since these adrenal steroids reach peak levels in the second decade of life and then gradually decline [32]. Moreover, high plasma DHEA concentrations correlate with longevity and survival in men but not in women [33]. DHEA may have beneficial effects on inflammation, cell growth, oxidative stress, carcinogenesis and atherosclerosis, possibly via inhibi- 
tion of glucose-6-phosphate dehydrogenase [34]. However, longer-term restriction of calories has been reported to leave plasma DHEA(S) concentrations unaffected in humans [31], and DHEAS supplements fail to extend lifespan or prevent chronic disease in rodents [35]. Therefore, the biological significance of the rise of plasma DHEA level we observed is uncertain. We did not find an effect of the prolonged fast on cortisol levels, which may have been caused by the relatively high levels of cortisol at baseline, perhaps induced by stress due to the insertion of the intravenous catheter.

Exposure to odorous and visual food cues did not modify any of the above-mentioned metabolic and neuroendocrine adaptations to a prolonged fast. It may be that olfactory cues do not impact on neuroregulatory mechanisms in humans. The olfactory system in higher mammals, including humans, comprises the olfactory bulb, the orbitofrontal cortex and the hypothalamus among other tertiary relay stations. A small number of in vivo fMRI studies show that odorous stimuli can alter hypothalamic neuronal activity in humans [6]. In our study, the presence of olfactory stimuli during fasting did not alter hypothalamic neuronal activity in response to a subsequent oral glucose load (during which no visual or odorous cues were offered). Although intuitively unlikely, the exposure time ( $9.5 \mathrm{~h} /$ day in total) we used may have been too short or the intensity of the odors may have been too weak to bring about significant effects. Furthermore, the impact of fasting on the regulation of metabolism may be too strong to allow significant effects of modulatory processes of lesser power. Indeed, the evidence in lower organisms indicates that olfactory cues restrain the beneficial corollaries of calorie restriction, not total starvation. Accordingly, Drosophila species were calorie restricted, not starved, in the study by Libert et al. [3]. In yeast (Saccharomyces cerevisiae), severe calorie restriction engages regulatory pathways to extend life span fun- damentally different from those activated by more modest restriction [36, 37]. In analogy, in humans (obese humans; we are unaware of studies in lean humans), modest restriction of calories does not affect insulin action in the short term [38], whereas total fasting considerably hampers insulin action in a similar time frame [39], suggesting that the degree of dietary restriction differentially affects regulatory mechanisms in humans as well. Thus, in contrast to calorie restriction, fasting may be too strong a stimulus for metabolic adaptation to allow olfactory cues to modulate the changes.

In conclusion, prolonged fasting changes the hypothalamic neuronal response to glucose ingestion in healthy normal-weight humans, which may guide postprandial endocrine and metabolic adaptations to nutrient deprivation that are meant to shunt any incidentally consumed carbohydrates towards the brain. Indeed, 60 h of total nutrient deprivation elicits profound glucose intolerance, most likely because it hampers insulin action. Prolonged fasting also downregulates the pituitarygonadal and -thyroid axes, probably to appropriately adapt fecundity and energy expenditure to nutrient scarcity. Food-related olfactory cues do not modulate these neuroendocrine and metabolic adaptations to a prolonged fast in humans.

\section{Acknowledgments}

We would like to thank Ron Wolterbeek for his advice on statistical analyses. Furthermore, we would like to thank Bep LadanEygenraam for her practical assistance and Bart Ballieux for his help with chemical analyses. Financial support was given by Roba Metals B.V., IJsselstein, The Netherlands. This work was also supported by the Center for Medical Systems Biology (CMSB), within the framework of the Netherlands Genomics Initiative (NGI/ NOW).

\section{References}

1 Fontana L, Partridge L, Longo VD: Extending healthy life span - from yeast to humans. Science 2010;328:321-326.

2 Bishop NA, Guarente L: Genetic links between diet and lifespan: shared mechanisms from yeast to humans. Nat Rev Genet 2007; 8:835-844.

-3 Libert S, Zwiener J, Chu X, Vanvoorhies W, Roman G, Pletcher SD: Regulation of Drosophila life span by olfaction and food-derived odors. Science 2007;315:1133-1137.
4 Alcedo J, Kenyon C: Regulation of C. elegans longevity by specific gustatory and olfactory neurons. Neuron 2004;41:45-55.

5 Keverne EB: The vomeronasal organ. Science 1999;286:716-720.

6 Wang J, Eslinger PJ, Smith MB, Yang QX: Functional magnetic resonance imaging study of human olfaction and normal aging. J Gerontol A Biol Sci Med Sci 2005;60:510-514.
Lam CK, Chari M, Lam TK: CNS regulation of glucose homeostasis. Physiology (Bethesda) 2009;24:159-170.

8 Schur EA, Kleinhans NM, Goldberg J, Buchwald D, Schwartz MW, Maravilla K: Activation in brain energy regulation and reward centers by food cues varies with choice of visual stimulus. Int J Obes (Lond) 2009;33: 653-661. 
\9 Matsuda M, Liu Y, Mahankali S, Pu Y, Mahankali A, Wang J, DeFronzo RA, Fox PT, Gao JH: Altered hypothalamic function in response to glucose ingestion in obese humans. Diabetes 1999;48:1801-1806.

10 Liu Y, Gao JH, Liu HL, Fox PT: The temporal response of the brain after eating revealed by functional MRI. Nature 2000;405:10581062.

-11 Vidarsdottir S, Smeets PA, Eichelsheim DL, van Osch MJ, Viergever MA, Romijn JA, van der Grond J, Pijl H: Glucose ingestion fails to inhibit hypothalamic neuronal activity in patients with type 2 diabetes. Diabetes 2007; 56:2547-2550.

-12 Simonson DC, DeFronzo RA: Indirect calorimetry: methodological and interpretative problems. Am J Physiol 1990;258:E399E412.

-13 Smith SM, Jenkinson M, Woolrich MW, Beckmann CF, Behrens TE, Johansen-Berg H, Bannister PR, De Luca M, Drobnjak I, Flitney DE, Niazy RK, Saunders J, Vickers J, Zhang Y, De Stefano N, Brady JM, Matthews PM: Advances in functional and structural MR image analysis and implementation as FSL. Neuroimage 2004;23(suppl 1):S208S219.

$\checkmark 14$ Woolrich MW, Jbabdi S, Patenaude B, Chappell M, Makni S, Behrens T, Beckmann C, Jenkinson M, Smith SM: Bayesian analysis of neuroimaging data in FSL. Neuroimage 2009;45:S173-S186.

15 Jenkinson M, Bannister P, Brady M, Smith S: Improved optimization for the robust and accurate linear registration and motion correction of brain images. Neuroimage 2002; 17:825-841.

$\checkmark 16$ Ogawa S, Lee TM, Kay AR, Tank DW: Brain magnetic resonance imaging with contrast dependent on blood oxygenation. Proc Natl Acad Sci USA 1990;87:9868-9872.

-17 Schwartz MW, Woods SC, Porte D Jr, Seeley RJ, Baskin DG: Central nervous system control of food intake. Nature 2000;404:661671.

18 Grove KL, Chen P, Koegler FH, Schiffmaker A, Susan SM, Cameron JL: Fasting activates neuropeptide $\mathrm{Y}$ neurons in the arcuate nucleus and the paraventricular nucleus in the rhesus macaque. Brain Res Mol Brain Res 2003;113:133-138.
19 McKibbin PE, McCarthy HD, Shaw P, Williams G: Insulin deficiency is a specific stimulus to hypothalamic neuropeptide Y: a comparison of the effects of insulin replacement and food restriction in streptozocin-diabetic rats. Peptides 1992;13:721-727.

20 van den Hoek AM, van Heijningen C, Schröder-van der Elst JP, Ouwens DM, Havekes LM, Romijn JA, Kalsbeek A, Pijl H: Intracerebroventricular administration of neuropeptide $\mathrm{Y}$ induces hepatic insulin resistance via sympathetic innervation. Diabetes 2008;57:2304-2310.

21 Hillebrand JJ, de Wied D, Adan RA: Neuropeptides, food intake and body weight regulation: a hypothalamic focus. Peptides 2002; 23:2283-2306.

22 Heijboer AC, Pijl H, van den Hoek AM, Havekes LM, Romijn JA, Corssmit EP: Gutbrain axis: regulation of glucose metabolism. J Neuroendocrinol 2006;18:883-894.

23 Smeets PA, Vidarsdottir S, de Graaf C, Stafleu A, van Osch MJ, Viergever MA, Pijl $\mathrm{H}$, van der Grond J: Oral glucose intake inhibits hypothalamic neuronal activity more effectively than glucose infusion. Am J Physiol Endocrinol Metab 2007;293:E754E758.

24 Webber J, Macdonald IA: The cardiovascular, metabolic and hormonal changes accompanying acute starvation in men and women. Br J Nutr 1994;71:437-447.

25 Chan JL, Heist K, DePaoli AM, Veldhuis JD, Mantzoros CS: The role of falling leptin levels in the neuroendocrine and metabolic adaptation to short-term starvation in healthy men. J Clin Invest 2003;111:1409-1421.

26 Mansell PI, Macdonald IA: The effect of starvation on insulin-induced glucose disposal and thermogenesis in humans. Metabolism 1990;39:502-510.

-27 Chan JL, Mietus JE, Raciti PM, Goldberger AL, Mantzoros CS: Short-term fasting-induced autonomic activation and changes in catecholamine levels are not mediated by changes in leptin levels in healthy humans. Clin Endocrinol (Oxf) 2007;66:49-57.

28 Leibel RL, Rosenbaum M, Hirsch J: Changes in energy expenditure resulting from altered body weight. N Engl J Med 1995;332:621628.

29 Speakman JR, Westerterp KR: Associations between energy demands, physical activity, and body composition in adult humans between 18 and 96 y of age. Am J Clin Nutr 2010;92:826-834.
30 Bergendahl M, Aloi JA, Iranmanesh A, Mulligan TM, Veldhuis JD: Fasting suppresses pulsatile luteinizing hormone (LH) secretion and enhances orderliness of $\mathrm{LH}$ release in young but not older men. J Clin Endocrinol Metab 1998;83:1967-1975.

31 Cangemi R, Friedmann AJ, Holloszy JO, Fontana L: Long-term effects of calorie restriction on serum sex-hormone concentrations in men. Aging Cell 2010;9:236-242.

32 Orentreich N, Brind JL, Rizer RL, Vogelman $\mathrm{JH}$ : Age changes and sex differences in serum dehydroepiandrosterone sulfate concentrations throughout adulthood. J Clin Endocrinol Metab 1984;59:551-555.

33 Mazat L, Lafont S, Berr C, Debuire B, Tessier JF, Dartigues JF, Baulieu EE: Prospective measurements of dehydroepiandrosterone sulfate in a cohort of elderly subjects: relationship to gender, subjective health, smoking habits, and 10-year mortality. Proc Natl Acad Sci USA 2001;98:8145-8150.

34 Schwartz AG, Pashko LL: Dehydroepiandrosterone, glucose-6-phosphate dehydrogenase, and longevity. Ageing Res Rev 2004;3:171-187.

- 35 Pugh TD, Oberley TD, Weindruch R: Dietary intervention at middle age: caloric restriction but not dehydroepiandrosterone sulfate increases lifespan and lifetime cancer incidence in mice. Cancer Res 1999;59:16421648.

36 Lin SJ, Defossez PA, Guarente L: Requirement of NAD and SIR2 for life-span extension by calorie restriction in Saccharomyces cerevisiae. Science 2000;289:2126-2128.

- 37 Kaeberlein M, Powers RW 3rd, Steffen KK, Westman EA, Hu D, Dang N, Kerr EO, Kirkland KT, Fields S, Kennedy BK: Regulation of yeast replicative life span by TOR and Sch 9 in response to nutrients. Science 2005;310: 1193-1196.

38 Jazet IM, Pijl H, Frolich M, Romijn JA, Meinders AE: Two days of a very low calorie diet reduces endogenous glucose production in obese type 2 diabetic patients despite the withdrawal of blood glucose-lowering therapies including insulin. Metabolism 2005;54: 705-712.

- 39 DeFronzo RA, Soman V, Sherwin RS, Hendler R, Felig P: Insulin binding to monocytes and insulin action in human obesity, starvation, and refeeding. J Clin Invest 1978;62: 204-213. 